\title{
Prevention of Peri-Anesthetic Hypothermia by Regulating Cutaneous Micro-Circulation: a Rodent Model
}

\author{
Nachum Nesher, Inna Frolkis, Amir Ganiel, Yanai Ben-Gal, Yosef Paz, Kramer Amir, Dimitri Pevni \\ Department of Cardiothoracic Surgery, Sourasky Medical Center, Sackler Faculty of Medicine, Tel-Aviv University, Israel.
}

\begin{tabular}{l} 
ARTICLE INFO \\
\hline Article history: \\
Received on: $30 / 12 / 2014$ \\
Revised on: 22/02/2015 \\
Accepted on: 14/03/2015 \\
Available online: $27 / 06 / 2015$ \\
\hline Key words: \\
Hibiscus sabdariffa calyces, \\
Roselle, anthocyanins, \\
colouring agents, colour \\
value.
\end{tabular}

\begin{abstract}
Objectives: Patients undergoing surgery involving general anesthesia often suffer from hypothermia, which is associated with various complications. External heating and warming are used to combat hypothermia throughout surgery; however their effectiveness is often limited. In this study we investigated a unique approach utilizing a natural physiological mechanism; protective cutaneous vasoconstriction for reducing peri-anesthetic hypothermia. The concept is based on the dermal application of a cream with vasoconstrictive alpha adrenergic agonist properties designed for this purpose; "thermal cream" (TC). Topical application of the cream was tested in a rodent model. The aim of the present study was to test the efficacy of a topical vasoconstrictive application in reducing peri-anesthetic hypothermia.

Materials and Methods: Adult Wistar rats were divided into two experimental arms: application of thermal cream was compared to application of a base cream without any active compound (control) under the same conditions. The rats were exposed to an ambient temperature of $21^{\circ} \mathrm{C}$. Their skin and core temperatures were measured and compared before, during and after anesthesia.

Results: The rats underwent rapid hypothermia immediately after the induction of anesthesia. The rats treated with thermal cream had a significantly higher core body temperature than the controls throughout the entire anesthetic period. This difference was even greater when the cream had been applied before the animal underwent anesthesia.

Conclusions: The thermal cream was effective in attenuating peri-anesthetic-induced hypothermia in a rodent model. It was easy to apply with no immediate mortality or other major adverse events.
\end{abstract}

\section{INTRODUCTION}

Hypothermia is defined as a rapid reduction in body temperature below $36^{\circ} \mathrm{C}$ and is often observed during surgery under general anesthesia (Sessler, 2008) especially during major operation such as any cardiac surgery (Nesher et al., 2003). Hypothermia may result in significant complications, such as arrhythmias, infections, cardiac stress and coagulopathies (Nesher et al., 2003, Reynolds et al., 2010). The responses to cold ambient temperature and the complications associated with such exposure are usually mitigated by factors associated with

\footnotetext{
* Corresponding Author

Nachum Nesher, Department of Cardiothoracic Surgery, Sourasky Medical Center, 6 Weizman St., Tel-Aviv, 64239, Sackler Faculty of Medicine, Tel-Aviv University, Israel. Telephone: +972-3-697-3322

Fax: +972-3-697-4439, Email: nesher61@ gmail.com
}

heat production and heat loss, including the severity and duration of cold stimuli, extension of surgical procedures, magnitude of the metabolic response, and others (Kurtz 2008, Kurz et al., 1995). Vasoconstriction and shivering are the main human mechanisms which are activated to prevent falling of body temperature following exposure to cold ambient temperature (Iampietro et al., 1969, Jacobs et al., 1994). These mechanisms are inhibited by general anesthesia (Sessler 2008). Thus, the inevitable consequences of cold insult can result in a somewhat uncontrolled drop in body temperature and high probability of hypothermia (Reynolds et al., 2010). Both external heating and warmed intravenous solutions have been used to re-warm the patient throughout the anesthetic period. However these technologies are often characterized by unpredictable and uncontrolled efficacy (Nesher $\mathrm{N}$ et al., 2003), a need for re-fitting, expensive and bulky storage and interference with the surgical field (Nesher et al., 2003, Hynson and Sessler 1992). 
We propose a concept that relies on endogenous physiological response (protective vasoconstriction) to an external cold exposure in order to reduce or even prevent peri-anesthetic hypothermia. This new modality is based on application of a topical cream which is a reformulation of a known therapeutic vasoconstricting alpha-adrenergic substance.

We tested this concept using an experimental immersion of epinephrine, a potent alpha receptor agonist, mixed with an inert basic cream and ethylene glycol as a penetration enhancer. The cream was applied in different concentrations as will be described. The cream aims to activate the body's natural protective response to a cold environment, which is mediated by a direct alpha adrenergic response causing vascular vasoconstriction which results in attenuation of heat convection. The cream is best applied just prior to induction of anesthesia in order to attenuate hypothermia.

The described concept was tested in vivo on rodents as part of proof of concept.

Adult Wistar rats (350-380 g) were chosen as an in vivo mammalian model to test the concept. All experiments were approved by the Tel-Aviv University Animal Welfare Committee. Every effort was made to minimize the number of required animals and their suffering. The animal model that would best reflect the human reaction to hypothermia was chosen after reviewing the results of applying hypothermic conditions on shaved or unshaved animals at the time of anesthesia.

The animals were placed inside an environmental chamber priested to $21^{\circ} \mathrm{C}$ in order to create an operating theater like conditions.

Both skin and core body temperatures were measured and recorded at the preselected time intervals as described below. The impact of different concentrations of the active component of the paste on hypothermic animals was also tested and compared to a control group.

\section{MATERIAL AND METHODS}

\section{The study was conducted as described in Figure 1.}

The animals were divided into two experimental arms: application of TC few minutes after the animals were anesthetized and application of base cream without any active compound (control) under the same experimental conditions. They all underwent identical anesthesia consisting of intraperitoneal injection of ketamine $(90 \mathrm{mg} / \mathrm{kg}$, Vetmarket, Israel) together with xylazine $(10 \mathrm{mg} / \mathrm{kg}$, Vetmarket, Israel). The thermal cream was a reformulation of epinephrine, mixed with a base cream containing the penetration enhancer propylene glycol. Core and skin temperatures were monitored throughout the study period at precise pre-selected time intervals, i.e., immediately after induction of anesthesia (baseline), and at 20, 40 and 60 minutes following the administration of anesthesia. After discontinuation of anesthesia the animals were allowed to wake up. Their core and skin temperatures were monitored at two, three, and four hours post-induction of anesthesia. Core temperature was measured by a rectal probe (Subcue Datalog-gers, Calgary, AB, Canada). After defining subanesthetic hypothermia in unshaved rodents exposed to an ambient temperature of $21^{\circ} \mathrm{C}$; (Group A - Figure 1), other 40 (completely shaved rats) were divided equally into four groups (Group B1-4). The thermal cream was applied over their entire shaved body as follows:

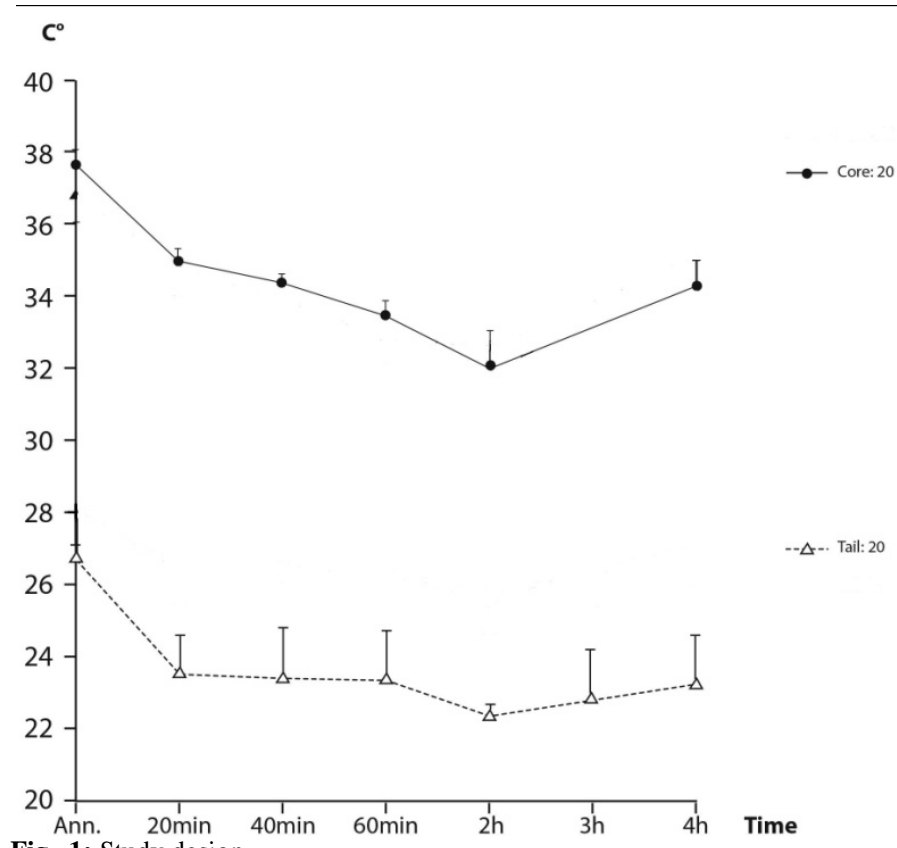

Fig. 1: Study design.

Group B1: application of base cream (propylene glycol 10\% penetration enhancement) without any active compound (control group).

Group B2: application of $7.5 \mathrm{mg} / \mathrm{ml}$ active compound in the same base cream

Group B3: application of $30 \mathrm{mg} / \mathrm{ml}$ active compound in the same base cream

Group B4: application of $60 \mathrm{mg} / \mathrm{ml}$ active compound in the same base cream

They were all exposed to an ambient temperature of $21^{\circ} \mathrm{C}$ as had been discussed above.

The next step of the study was conducted following the results obtained from the previous step, using non-shaved rats only (Groups C1-4, Figure 1). Thermal cream was applied on the nonhairy regions of the animal's body.

The aim of this arm was to investigate the influence of covering the non-hairy parts of the body with artificial cover (in non-shaved rats) on temperature profiles. This was compared to the temperature profiles obtained from non covered animal and those in whom these parts were covered with either thermal cream or basic cream.

Four groups of unshaved rats $(n=10$ each) were compared. The tail, feet and ears of the rats from the first group (Group C1) were covered by cotton while rats from the second group (Group C2) had no similar cover. In the other two groups, the tails and other non-hairy parts were covered by either thermal 
cream (containing 6\% $(60 \mathrm{mg} / \mathrm{ml})$ active component immersed in $10 \%$ propylene glycol), (Group C3) or with the basic non-active cream in the fourth group (Group C4). The tail temperature, abdominal skin (under the fur) and core temperatures were recorded at the preselected time intervals.

The last step was to define what should be the exact timing of TC application and the amount of penetration enhancer needed to maximizes its efficiency.

The penetration enhancer (PE) was doubled (to 20\%), and the thermal cream was applied twice to the tail ears and feet, (first $\sim 30$ minutes before induction of anesthesia and then immediately after the induction) (Group D). Results obtained from this study were compared to those from the previous step; i.e. $\mathbf{C 4}$ - covered by the basic cream only and C3 - study cohort immersed in 10\% penetration enhancer.

\section{Statistical Analysis}

Results are presented as the mean \pm SD. All temperature measurements were subjected to two-way analysis of variance (ANOVA) with repeated measures. This design includes one between-subject factor (the experimental group) and one withinsubject factor (the time of measurement). Whenever a significant time trend was demonstrated, we used contrast analysis to compare each measurement to its successive one (SIMPLE; SPSS Inc., Chicago, IL). The Wilcoxon test was performed to compare the measurements of temperature between separate time points in each experimental study. A power analysis suggested that a sample size of 10 is required to achieve a power of 0.8 testing at $\mathrm{p}=0.05$.

\section{RESULTS}

Definition and verification of subanesthetic hypothermia in rodents (Group A, $n=10$ ): Exposure to cold ambient resulted in rapid dropped of core body temperature as it had from the induction of anesthesia: $38^{\circ} \mathrm{C} \pm 1.2^{\circ}$ to $35.56^{\circ} \mathrm{C} \pm 0.26$ (Figure 2). There was no significant difference between the core and the skin (under the fur) however the skin temperature profile measured at the tail was significantly higher from the one measured under the fur. The core and the skin temperatures started to rise after three hours post-anesthesia (full awakened state). After confirming the definitions and verifying the development of rapid hypothermia caused by exposure to cold ambient the next step was evaluated.

Applying different compositions of thermal cream (Group B, $\mathrm{n}=40)$ :

Core and skin temperatures dramatically dropped from the induction until four hours post-anesthesia. The core body temperature of all animals declined within 20 minutes to $28^{\circ} \mathrm{C} \pm$ $1.1^{\circ}$. There was no difference in the temperature profiles of all 4 groups (ANOVA $P=\mathrm{NS}$ ). There was no difference between the skin and core body temperatures. Increasing the amount of active compound in the cream had no influence on hypothermic reaction in shaved rat's model. Covered versus not covered rats (Group C, $\mathrm{n}=40$ ): As had been observed in our above-cited investigations, transferring the rats into the $20^{\circ} \mathrm{C}$ chamber caused immediate hypothermia. There was, however, a difference in the body temperature profile between the covered and non-covered hairy rats. Core body temperature was $1.5^{\circ} \mathrm{C} \pm 0.8^{\circ}$ lower in the covered group $(\mathrm{C} 1)$ as measured from 20 minutes post-induction compared with non-covered $\operatorname{group}(\mathrm{C} 2$, Fig. 3). There was a similar difference in the skin temperature profile measured on the abdomen.

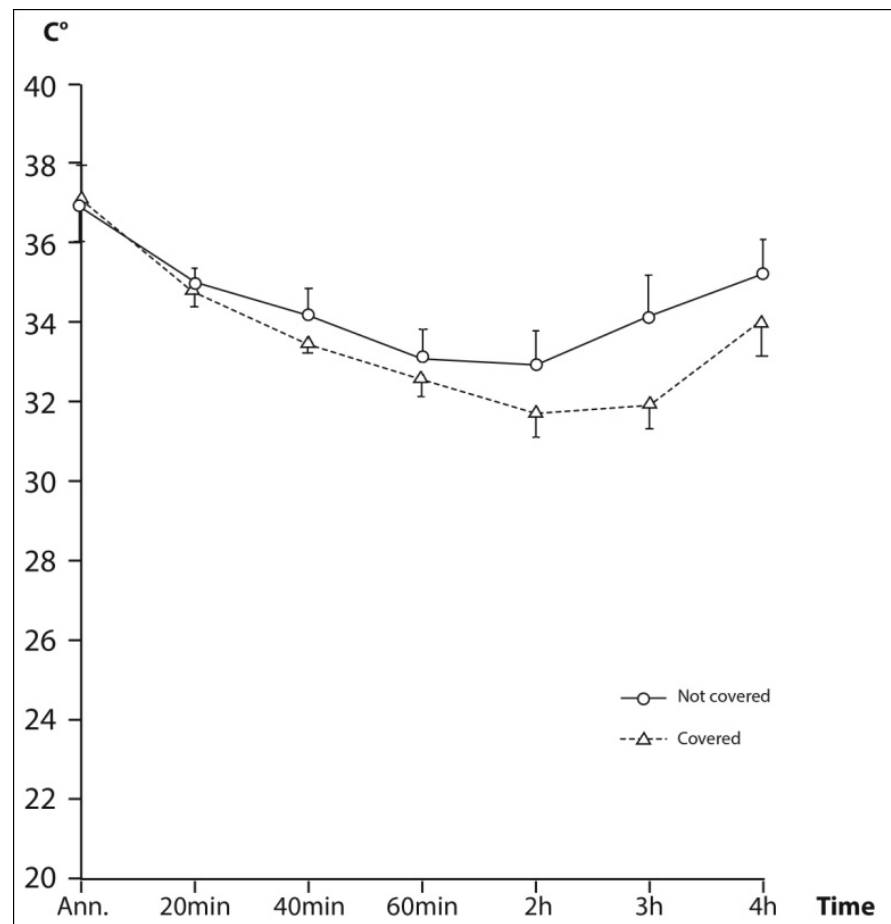

Figure 2. Core and Tail temperatures in a cold ambient. Non-shaved rats.

There was a consistent higher core body temperature, $\geq 1^{\circ} \mathrm{C}$ in the thermal cream group (C3) compared to the control group (C4) observed from 40 minutes post anesthesia (ANOVA $P$ $>0.01$ ) (Fig. 4 black lines). Skin temperature measured at the tail yielded an opposite results: the group applied with thermal cream had a lower tail temperature of $\sim 2^{\circ}$ compared to the control (Fig. 4 black lines). There was no difference in abdominal skin temperature (ANOVA $P=\mathrm{NS}$ ).

The effect of the thermal cream on the core body temperature started 45 minutes after the induction of anesthesia and reached a level of significance one hour after the induction of anesthesia. (Fig. 4 Top) Its peak effect lasted for almost four hours after induction of anesthesia.

Timing of application and the amount of penetration enhancer needed to maximizes its efficiency. (Group D, n=10):

A wider and an earlier difference in core body temperature between the study cohort and the control (basic cream only) was observed. There was a significant difference in core body temperatures between the $20 \% \mathrm{PE}$ group and the control group starting few minutes after anesthesia, reaching level of significance at 20 minutes after induction. $\left(37^{\circ} \pm 0.6^{\circ} \mathrm{C}\right.$ vs. $35^{\circ} \pm$ $0.8^{\circ} \mathrm{C}$ respectively, ANOVA $\left.P<0.0001\right)$. This difference lasted for the entire study period (four hours). There was also a 
significant difference in core body temperature between the $20 \%$ $\mathrm{PE}$ group, in which the cream was applied twice and the $10 \% \mathrm{PE}$ group, when the cream was applied only once. This difference started immediately and reached significance at 20 minutes after induction of anesthesia $\left(37^{\circ} \pm 0.4^{\circ} \mathrm{C}\right.$ vs. $35^{\circ} \pm 0.7^{\circ} \mathrm{C}$ respectively, ANOVA , $P<0.0001)$. This difference lasted for only one hour and then disappeared, (Fig. 4 gray line).

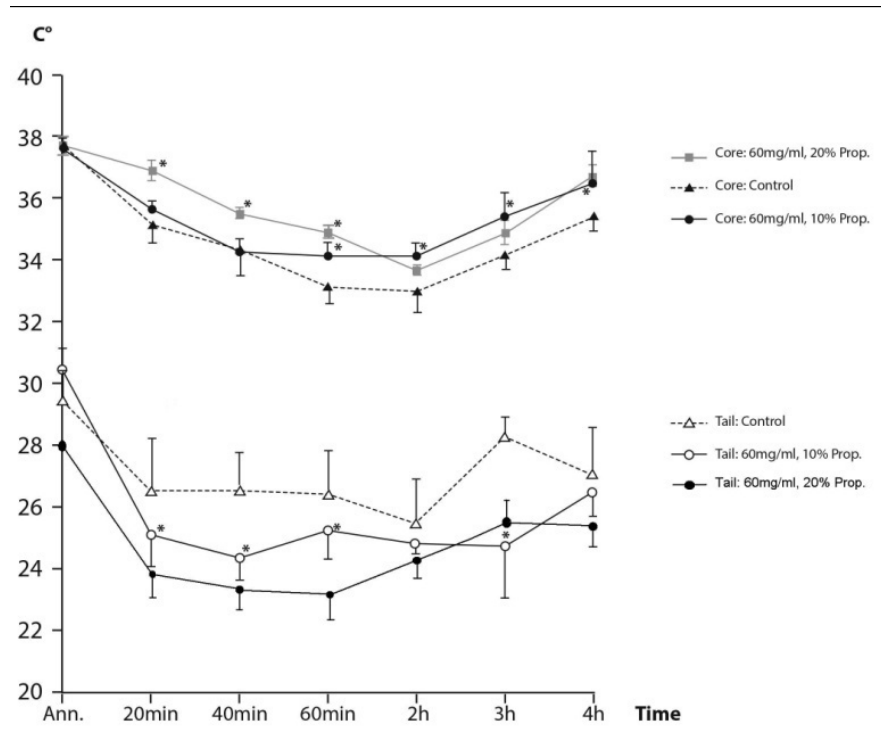

Fig. 3: Effect of covered vs. uncovered tails on the core temperature in nonshaved rats.

\section{DISCUSSION}

We have created a reliable animal model for defining subanesthetic hypothermia and evaluating the influence of different concentrations of the thermal cream, composed of an alpha adrenergic receptor agonist, on body temperature profiles, under these conditions. The results of the current study demonstrated that thermal cream was effective in attenuating perianesthetic-induced hypothermia in a rodent model. There was no mortality or major immediate untoward event even though a relatively large amount of the active substrate having been applied. The absence of any difference between the skin and core temperatures in the shaved rats suggested a lack of peripheral thermoregulatory activity at the skin under the fur. This was further supported by the fact that no difference was observed in the shaved rats reactions to the active vasoconstrictive cream of any composition. Application of the thermal cream solely on the nonhairy regions of unshaved rats, however, may be explained by cutaneous capillary vasoconstriction which results in a significant attenuation of hypothermia. A large difference between tail's skin and core body temperatures (at the non-hairy parts), in non-shaved rats, supports a major thermoregulatory role for these parts of a rat's body. Since the surface area of the tail is smaller than the entire body surface and the skin of the tail is thicker, we concluded our study evaluating the influence of doubling the amount of penetration enhancer and the timing of application. There was a significant difference in core body temperature between the study and the control group from 20 minutes after the induction of anesthesia. This difference, which lasted for only one hour after application and then disappeared, is likely due to the faster and more efficient absorption of the active component with the double penetration enhancer formulation. Body temperature is regulated through a balance between heat generation and its dissipation and it is modulated among most mammals usually in a very narrow range $\left(35^{\circ} \mathrm{C}-40^{\circ} \mathrm{C}\right)$ and within which physiological function is optimal (Pozos et al., 1996). Clinical hypothermia occurs when core body temperature falls below $36^{\circ} \mathrm{C}$. Myocardial function is depressed and ventricular fibrillation may show up as the greatest hazard associated with hypothermia (Hervey 1993). Nevertheless survival and outcomes are linked with the capacity of treating effectively or totally prevent perioperative hypothermia (Sessler 2008, Nesher et al., 2003, Reynolds et al., 2010). Convective heat loss is a dominant mechanism of thermal energy transfer in unprotected cold ambient, particularly during anesthesia (Romanovsky 2007). Lower skin temperature stimulates thermoreceptors, and the resultant afferent flow elicits autonomic homoeostatic responses (thermogenesis and vasoconstriction) regulating the body temperature (Kellogg 2006, Richardson and Shepherd 1991, Frank et al., 1997). The most powerful responses occur when both superficial and deep thermoreceptors are cooled simultaneously, as in any major surgery, although thermo-effector activation can also occur as a result of peripheral cooling alone like of exposure to cold ambient temperature even before commencement of surgery (Kellogg 2006). The responses to ambient cold temperature are moderated by the severity and duration of cold stimuli, the extended duration of surgical procedures, the magnitude of metabolic response, and other individual characteristics (Kurtz 2008). Thermoneutrality exists when only cutaneous vascular responses are recruited to regulate body temperature. At rest, the ambient temperature for thermoneutrality varies as a function of characteristics of the medium (air $=23^{\circ} \mathrm{C}-26^{\circ} \mathrm{C}$ and water $=34^{\circ} \mathrm{C}-36^{\circ} \mathrm{C}$ ). The lower critical temperature that defines the lower extreme of thermoneutrality and it is the temperature below which shivering is initiated. At thermoneutrality heat fluxes are controlled and temperature is regulated via changes in cutaneous vascular tone. When ambient temperature drops below the lower critical temperature, autonomic skin vasoconstriction and metabolic energy transformation (thermogenesis) are initiated, both of which represent the primary cold-effector responses (Webb and Shepard 1968). Cutaneous circulation is a major effector for mammalian thermoregulation. Local cooling of the skin causes a local temperature-dependent vasoconstriction which is totally dependent on intact sympathetic activity. All thermoregulatory mechanisms, i.e., shivering, thermogenesis and vasoconstriction, are inhibited by general anesthetics via multiple mechanisms (Reynolds et al., 2010). However, many of them still remain undefined. During cold stress, reduced temperature leads to cutaneous vasoconstriction through combined neural and local mechanisms. Under normothermia skin blood flow averages about $10 \%$ or more of cardiac output and it is reduced to near zero during 
hypothermia. Work done over the last several decades has suggested a role for an alpha-adrenergic response in causing cutaneous vasoconstriction in response to local skin temperature insult (Kellogg 2006). Our results may be explained by these reports. The cutaneous blood flow response varies across different body regions (Hensel H 1981), largely due to local variations in vasoconstrictor control, with major differences between the apical (non-hairy) and the non-apical (hairy) regions. The rat's apical regions (feet, hands, lips, nose, ears and tail) are innervated solely by adrenergic sympathetic nerves; therefore thermoregulatory reflexes in these regions are mediated by adrenergic vasoconstrictor tone. The non-apical regions, limbs and trunk, are under dual control of the adrenergic, vasoconstrictor system and an active vasodilator system for which the neurotransmitter has yet to be isolated (Kellogg 2006, Roddie 2003). Therefore, the thermoregulatory reflex is minor at these regions, probably due to this combined effect of both sympathetic and parasympathetic activity. A mammal's, distal skin regions, particularly the hands, feet (Roddie 2003), and ears (Prichard and Daniel 1956), contain specialized networks of arteriovenous anastomoses which supply blood to the venous plexus directly from small arteries. These anastomoses play an important role in temperature regulation, since their synchronous closing, caused by efferent sympathetic impulse bursts (Hensel 1981, Roddie 2003), is linked to heat balance (Burton 1939). This regulation was thought to be predominantly unique to humans, however Chotani and Flavahan (Chotani et al., 2000) explored this role of adrenergic receptors in the mouse tail and found some activity similar to that described above (Hensel 1981, Roddie 2003, Prichard and Daniel 1956, Burton 1939).

\section{CONCLUSIONS}

Application of thermal cream attenuated peri-anesthetic hypothermia in a rodent model. Our study supports the assumption that the thermal cream caused a direct reduction of coutaneous peripheral blood flow due to local vasoconstriction. By doing so, it may promote the first step in modulating heat loss, and, as a result, it may attenuate undesired, peri-anesthetic hypothermia. The topically applied vasoconstrictive formulation caused delayed cooling of deeper tissues by obstructing conductive pathways for heat loss. These findings support a concept by which this physiological stimulus can be used to prevent hypothermia and therefore should be tested in the future on larger animal models.

\section{Study limitations}

This is an observational study which does not supported by any molecular physiology yet. Rodent's hemodynamic response was not detected in an attempt to avoid further discomfort. Meticulous hemodynamic monitoring should be applied in a larger size animal model.

\section{Abbreviations}

TC - Thermal cream, PE - Penetration enhancer.

\section{REFERENCES}

Sessler DI. Temperature monitoring and perioperative thermoregulation. Anesthesiology, 2008; 109:318-38.

Nesher N, Zisman E, Wolf T, Sharony R, Bolotin G, David D, Uretzky G, Pizov R. Strict thermoregulation attenuates myocardial injury during CABG surgery as reflected by reduced levels of cardiac-specific Troponin I. Anesthesia \& Analgesia, 2003; 96: 328-35.

Reynolds I, Beckmann J, and Kurz A. Perioperative complications of hypothermia. Best Pract Res Clin Anaesthesiol, 2010; 22:645-57.

Kurtz A. physiology of thermoregulation. Best Pract Clin Anesthesiol, 2008; 22:627-44.

Kurz A, Sessler DI, Christensen R, and Dechert M. Heat balance and distribution during the core-temperature plateau in anesthetized humans. Anesthesiology, 1995; 83:491-9.

Iampietro PF, Vaughan JA, Goldman RF, Kreider MB, Masucci F, and Bass DE. Heat production from shivering. J Appl Physiol, 1969; $15: 632-4$.

Jacobs I, Martineau L, and Vallerand AL. Thermoregulatory thermogenesis in humans during cold stress. Exerc Sports Sci Rev, 1994; 22:221-50.

Hynson JM, and Sessler DI. Intraoperative warming therapies: a comparison of three devices, J Clin Anesth 1992; 4:194-9.

Pozos RS, Iaizzo PA, Danzl DF, and Mills WT Jr. Limits of tolerance to hypothermia. In: Fregly MJ, Blatteis CM, eds. Handbook of physiology. II. The thermal environment. New York: Oxford; 1996:55775.

Hervey GR. Proceedings: Physiological changes encountered in hypothermia. Proc R Soc Med; 1993; 66:1053-8.

Romanovsky AA. Thermoregulation: some concepts have changed. Functional architecture of the thermoregulatory system. Am J Physiol Regul Inter Comp Physiol, 2007; 292:R37-46.

Kellogg DL Jr. In vivo mechanisms of cutaneous vasodilatation and vasoconstriction in humans during thermoregulatory challenges. J Appl Physiol, 2006; 100:1709-18.

Richardson D, and Shepherd S. The cutaneous microcirculation of the forearm in young and old subjects. Microvasc Res, 1991; 41:84-91.

Frank SM, Fleisher LA, Breslow MJ, Higgins MS, Olson KF, Kelly S, and Beattie C. Perioperative maintenance of normothermia reduces the incidence of morbid cardiac events. A randomized clinical trial. JAMA, 1997; 277:1127-34.

Webb PM, and Shepard JT. Responses of superficial limb veins of the dog to changes in temperature. Circ Res, 1968; 22:737-46.

Hensel $H$. Thermoreception and temperature regulation. Monographs of the Physiological Society number 38. London: Academic Press, 1981; 46:213-17.

Roddie IC. Sympathetic vasodilatation in human skin. J Physiol (Lond), 2003; 548:336-7.

Prichard ML, and Daniel PM. Arteriovenous-anastomoses in the human external ear. J Anat, 1956; 90:309-17.

Burton AC (1939). The range and variability of the blood flow in the human finger and the vasomotor regulation of body temperature. Am J Physiol 127:437-53.

Chotani MA, Flavahan S, Mitra S, Daunt D, and Flavahan NA. Silent alpha(2C)-adrenergic receptors enable cold-induced vasoconstriction in cutaneous arteries. Am J Physiol Heart Circ Physiol, 2000; 278:H1075-83.

\section{How to cite this article:}

Nachum Nesher, Inna Frolkis, Amir Ganiel, Yanai Ben-Gal, Yosef Paz, Kramer Amir Dimitri Pevni. Prevention of Peri-Anesthetic Hypothermia by Regulating Cutaneous Micro-Circulation: a Rodent Model. J App Pharm Sci, 2015; 5 (06): 104-108. 